DOI $: 10.22199 / \mathrm{S} 07160917.1982 .0002 .00004$

\title{
ISAAC NEWTON (1642-1727)
}

\author{
Ma. DRINA ROJAS MEDAR
}

HUGO RODRIGUEZ CORTEZ

(alumnos licenciatura)

Matemático, físico y astrónomo inglés, una de las grandes figuras de la historia de la ciencia, nació en Woolsthorpe, cerca de Grantham, en Lincolnshire, el 25 de Diciembre de 1642 y murió en Nansington el 20 de Mayo de 1727. Hijo de un labrador acomodado, muerto antes de su nacimiento, su madre Ana Ayscough, se casó poco después con Bernabé Smyth, rector de Nortwitham, y confió al pequeño Isaac, que entonces tenía tres años, a los cuidados de su abuela, quien lo envió a la escuela rural. A los doce años fué enviado a estudiar a la escuela de Grantham, hospedándose en casa del doctor Clark, un farmacéutico de la población. Allí paso tres años y no se distinguió por su afición al estudio, a excepción de las matemáticas, que parecía interesarle un poco; en cambio se mostró muy apasionado por la mecánica y construyó o ideó un gran número de ingenioso juguetes. Al mismo tiempo se ejercitaba en el dibujo llenando con sus compo- 
siciones las paredes de la habitación que ocupaba. Fue entonces, y en casa del Dr. Clark, donde conoció a la señorita Storay que le inspiró una profunda pasión y para la que guardó después su más tierna amistad. Viuda por segunda vez su madre (1657), lo llamó a su lado para que se encargase de la granja de Whodstorpe, pero mostró poca afición a la agricultura, entregándose de lleno a la lectura de obras de matemáticas y de física que le había facilitado el Dr. Clark.

Un tío materno, adivinando las verdaderas aptitudes y aficiones del joven Isaac, convenció a su madre que 1o enviara nuevamente a la Escuela de Grantham, donde estuvo dos años. En 1661 ingresó en el Trinity College de Cambridge, institución en la que se recibió cuatro años más tarde, aunque permaneció luego en la misma como miembro.

En el otoño de 1665, el esparcimiento de una gran plaga motivó que el Trinity College cerrara sus puertas por un año y medio. Hasta su reapertura permaneció en su ciudad natal. Durante aquellos dieciocho meses, sentó las bases de sus famosos descubrimientos tanto en matemáticas como en física: Teorema del binomio; Método de las fluxiones, fundamento del cálculo diferencial e integral; y descubrió que la fuerza de gravitación de la tierra mantiene a la luna en su órbita, si bien acerca de este hallazgo no publicó cosa alguna, hasta pasados casi veinte años.

Vuelto a Cambridge en 1667, sucedió a sus profesor Isaac Barrow en la cátedra de matemáticas que éste dictaba en el Trinity College, mientras que por otra par- 
te se dedicó a investigaciones sobre óptica que le condujeron a construir el primer telescopio de reflexión, invento que le valió pertenecer a la Sociedad Real, la institución científica inglesa de mayor nota. De esta misma época data sus descubrimiento de la causa que determina la dispersión de la luz blanca cuando la misma atraviesa un prisma. Enviado a la Sociedad Real el trabajo en que se consignaba tal hipótesis, se suscitaron controversias en el mundo científico de la época que a su vez impidieron a Newton a efectuar otros experimentos de óptica cuyos resultados compendió posteriormente en su libro "Optica o tratado de las reflexiones, refracciones, inflexiones y colores de la luz". (1704).

En 1687 apareció su obra capital: "Principios matemáticos de la filosofía natural", en la que expuso las leyes fundamentales de mecánica clásica así como su famosa teoría de la gravitación universal, que explica el movimiento de los planetas en el espacio. Esta obra valió gran renombre al autor, quien en 1695 fue nombrado Inspector de la Casa de la Moneda de Inglaterra, de la que en 1699 llegó a ser Director. En el mismo año la Academia de Ciencias Francesa lo incluyó entre sus ochos miembros extranjeros, y un año más tarde, la reina Ana de Inglaterra lo armaba caballero.

En 1701 renunció a 1 cátedra en Cambridge, y pasó a establecerse en Londres, donde en 1703 se lo acogió para presidir la Sociedad Real, cargo que mantuvo hasta su muerte. Los último años de este científico se vieron turbados por su mala salud $y$ por las controversias que sostuvo con Leibniz en cuanto a cuál de los dos correspondió el honor de haber creado el cálculo diferencial. 
En la actualidad se considera que Leibniz inventó esta rama de las matemáticas independiente de Newton.

\section{LA OBRA DE ISAAC NEWTON.}

Newton consideró las magnitudes matemáticas constituídas, no por partes arbitrariamente pequeñas, sino engendradas por un movimiento continuo. Así, las líneas no se engendran mediante suma de partes, sino por el movimiento continuo de puntos; las superficies, por el movimiento de líneas; los sólidos por el movimiento de superficies; los ángulos por la rotación de sus lados; los tiempos por el flujo continuo.

Consideró además que las cantidades engendradas, al crecer en tiempos iguales, resultan mayores o menores, según la velocidad mayor o menor con que crecen. Buscó un método para determinar la magnitud de las velocidades de los movimientos o de los incrementos con que se engendran. Llamando "Fluxiones" estas velocidades que incrementan el movimiento, y "Fluentes" las cantidades engendradas. Si $x$ es la cantidad variable, $\dot{x}$ la fluxión respectiva, o sea $1 \mathrm{a}$ velocidad de su variación, mientras que la variación misma (nuestra diferencial) será o $\dot{x}$, siendo o el instante de tiempo en el que se produce aquella variación.

La fluxiones las consideró, con aproximación arbitrariamente grande, como los incrementos de los fluentes engendrados durante iguales intervalos de tiempo arbitrariamente pequeños; más exactamente, son directamente proporcionales a los incrementos instantáneos de los fluentes y se pueden además, representar mediante líneas proporcio- 
nales a las mismas.

Si, por ejemplo el área del triángulo mixtilíneo $A B C$ y el área del rectángulo $A B D G$, son engendrados por el movimiento uniforme de las ordenadas $B C$ y $B D$ que avanzan a lo largo de la base $A B$, entones las fluxiones de estas área están entre sí como las ordenadas BC y BD que las engendran, porque estas ordenadas están entre sí como los incrementos instantáneos de las áreas.

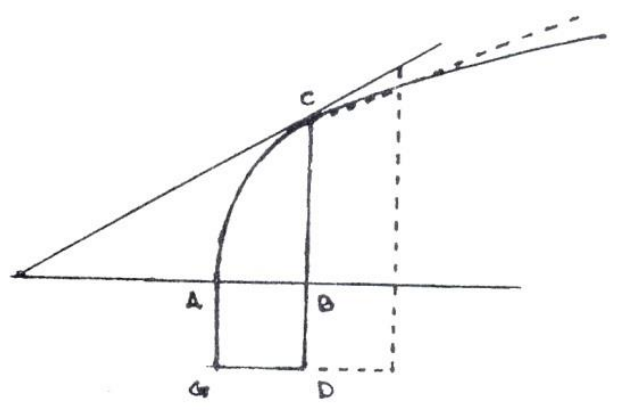

PROBLEMA. Si la cantidad $x$ fluye uniformemente, determinar la fluxión en la cantidad $x^{n}$.

SOLUCION. En el tiempo en que la cantidad $x$, al fluir se convierte en $x+o$, la cantidad $x^{n}$ se convierte en $(x+o)^{n}$ $y$, por tanto, de acuerdo con el método de las series infinitas, en:

$x^{n}+n o x^{n-1}+\frac{n^{2}-n}{2}$ oox $x^{n-2}+$ etc. y los incrementos o y

$n o x^{n-1}+\frac{n^{2}-n}{2}$ oox $x^{n-2}+$ etc. están entre sí como 1 a

$n x^{n-1}+\frac{n^{2}-n}{2} o x^{n-2}+e t c$.

Si el incremento desvanece, esa razón última será: $1: n x^{n-1}$. 
Con consideraciones semejantes, mediante el método de las primeras y últimas razones se pueden determinar las fluxiones de las líneas, sean rectas o curvas, en casis cualesquiera, así como las fluxiones de superficies, de ángulos, o de cualquier otra cantidad.

La $o$ que introduce Newton en los párrafos precedentes, se parece extrañamente a un cero, pero en las demostraciones adopta un papel ambiguo, pués después de haber actuado como cantidad no nula, de pronto se anula. Si se exceptúa esta anomalía, lo restante del algoritmo newtoniano coincide con el actual y los resultados son correctos.

Entre estos resultados figura como contribución importante la inversión del problema de la cuadratura. En efecto, Newton invirtiendo el proceso habitual de determi-nar el área encerrada por una curva partiendo de la orde- nada de la misma, parte de la expresión del área y apli- cándole el método utilizado para determinar la tangente a una curva plana, llega a la ordenada, comprobando así, que los problemas de la tangente y de la cuadratura son problemas inversos $y$, por tanto, en el fondo la misma cuestión. Esta demostración constituye la clave de bóve- da que cierra el edificio del cálculo infinitesimal y per- mite reunir sus problemas, tratados en forma separada, en un solo haz.

\section{BIBLIOGRAFIA.}

1. Enciclopedia BARSA (1968)

2. Enciclopedia BRITANICA (1968)

3. Enciclopedia ESPASA CALPE (1954)

4. CALCULO INFINITESIMAL (1972) 